Conclusion Our survey identified stakeholder dissatisfaction, which has initiated plans for further needs-assessments and renovation. Our patients were largely indifferent towards the PAR environment, which was in contrast to parents and staff. Evidence suggests parental distress is associated with child distress during induction of anaesthesia, and it is therefore imperative that parents must be consulted in our re-design process which should focus on relieving distress of both parent and child.

\section{G404(P) CATCHING UP WITH THE 21ST CENTURY (THROUGH CHARITY!): IS A COMPUTERISED CLINICAL JOBS BOOK SUPERIOR TO A PAPER BASED JOBS DIARY?}

${ }^{1} \mathrm{C}$ Beatty, 'L Simpson, ${ }^{1} \mathrm{MS}$ Javed, ${ }^{2} \mathrm{MS}$ Javed, ${ }^{2} \mathrm{R}$ White, ${ }^{1} \mathrm{Z}$ Baacha, ${ }^{1} \mathrm{Z}$ Saleem. ${ }^{1}$ Department of Paediatrics, Walsall Hospitals NHS Trust, Walsall, UK; ${ }^{2}$ Applications Engineering Department, Silicon Labs, High Wycombe, UK

\subsection{6/archdischild-2018-rcpch.393}

Background With increasing workload and reducing length of stay, a robust system was needed to keep track of outstanding clinical jobs regarding patients whose early discharge was made possible by the on-site presence of senior decision makers. A paper based diary initially proved successful and results were presented as a $(\mathrm{P})$ at the $\mathrm{RCPCH}$ annual conference 2015. However, this system did not keep up with demands and posed several clinical governance challenges. Therefore, a computerised network based system (PaedsTMS) was developed with help of employees of a multinational technology firm as a charity project.

Aims To assess the effectiveness and quality of jobs recording and completion, before and after the introduction of PaedsTMS.

\begin{tabular}{|c|c|c|c|c|c|c|}
\hline & $\begin{array}{l}\text { Pre- } \\
\text { paedsTMS } \\
\text { audit } \\
(\mathrm{n}=31)\end{array}$ & & $\begin{array}{l}\text { Post- } \\
\text { paedsTMS } \\
\text { audit-1 } \\
(\mathrm{n}=27)\end{array}$ & & $\begin{array}{l}\text { Re- } \\
\text { audit-2 } \\
(n=30)\end{array}$ & \\
\hline & $\begin{array}{l}\text { No of } \\
\text { patients }\end{array}$ & $\begin{array}{l}\% \\
\text { achieved }\end{array}$ & $\begin{array}{l}\text { No of } \\
\text { Patients }\end{array}$ & $\begin{array}{l}\% \\
\text { achieved }\end{array}$ & $\begin{array}{l}\text { No of } \\
\text { Patients }\end{array}$ & $\begin{array}{l}\% \\
\text { achieved }\end{array}$ \\
\hline $\begin{array}{l}\text { Jobs added to } \\
\text { system }\end{array}$ & $30 / 31$ & 96 & $21 / 27$ & 78 & $26 / 30$ & 87 \\
\hline $\begin{array}{l}\text { Sufficient task } \\
\text { information } \\
\text { provided }\end{array}$ & $9 / 30$ & 30 & $19 / 21$ & 90 & $26 / 26$ & 100 \\
\hline $\begin{array}{l}\text { Task } \\
\text { Completed }\end{array}$ & $17 / 30$ & 57 & $21 / 21$ & 100 & $26 / 26$ & 100 \\
\hline $\begin{array}{l}\text { Task } \\
\text { completed by } \\
\text { due date }\end{array}$ & $\begin{array}{l}\text { Information } \\
\text { not } \\
\text { available }\end{array}$ & - & $13 / 20$ & 65 & $20 / 26$ & 77 \\
\hline
\end{tabular}

Methods PaedsTMS was introduced in June 2017 after an initial audit of the existing paper based system. The project was led by a registrar under guidance of a consultant paediatrician. A team of champions from medical, nursing and clerical background was assembled. Team discussions were done on messaging apps. Training was accomplished face to face and by video tutorial on YouTube. PaedsTMS was run parallel to the existing system for two weeks. Two audits were completed after introduction of PaedsTMS in July and September 2017. We looked at the percentage of tasks added to PaedsTMS, percentage of tasks added with sufficient clinical information, percentage of outstanding tasks completed and percentage of tasks completed within due date. The results were compared with pre-PaedsTMS introduction audit.

Results The re-audit showed significant improvements as depicted in table 1 below:

Conclusion The computerised system has significantly increased the quality of clinical details recorded with jobs as well as their timely completion whilst providing a clear audit trail to satisfy clinical governance standards.

\section{G405(P) SENIOR SAFETY WALK IN A DISTRICT GENERAL HOSPITAL}

${ }^{1}$ AK Khan, ${ }^{2}$ GM Mcguire, ${ }^{2}$ PM Morgan. 'Paediatrics, Daisy Hill Hospital, Southern Health and Social Care Trust, Newry, UK; ${ }^{2}$ Children and Young Pople Directorate, Southern Health and Social Care Trust, Newry, UK

\subsection{6/archdischild-2018-rcpch.394}

Background Senior safety walk (SSW) is a structured process to bring senior managers and front line staff together to have patient safety conversations with a purpose to mitigate service user/staff harm.

Aim To Introduce Senior Safety Walks in Paediatric Department:

- To demonstrate senior managers' commitment to quality and safety for staff and service users,

- To Increase staff engagement and develop a culture of open communication and

- To Identify, acknowledge and share good practice

Method PDSA Cycle used as QI methodology. A literature search was completed. SSW team members included a senior operation manager, senior clinician and admin person. Monthly SSWs scheduled. SSW toolkit developed including: MDT Communication frame, Sample guide for SSW discussion, Data collection templates and Impact measurements.

Impact measurements: Number of problems identified which were addressed within time period;Number of safety-based changes made by staff by per year;Number of compliments/ complaints received (outcome measure); and Response to staff safety culture survey (process measure).

Results

- Senior Safety Walks started in Feb 17.

- SSW Themes measured: 1)Leadership, 2)Communication and Team working, 3)Environment and Processes, 4) Patient/ carer feed back

- Each SSW Included following actions:

- Observation of Environment, Process and Leadership

- Staff discussion including feedback measures discussed and implemented

- Services user/Patient/carer discussion

\section{- Impact measurement results:}

- $85 \%$ of identified issues were resolved within agreed time frame.

- Multiple safety based changes made in the department.

- Number of complements received from services users during this period. 
- Staff survey findings: Improvements in understanding of patient safety measures. Also highlights staff satisfaction for senior managers involvement at front line issues.

- Service User feedback and actions taken :

'Ward too cold at night'- New children's unit due to commission in Dec 17. Have new climate controls

'Small/dated children play area'- Children's play area redesigned. 'Had to go home to get toothbrush and phone charger'- New

'Welcome Pack' developed.

\section{Conclusion}

- Senior safety walks is a valuable tool to engage senior managers and frontline staff in a meaningful discussion of patient safety concerns. It can also lead to and resolution of safety issues with agreed actions. This can also improve team working and safety culture of the organisation.

- SSW is resource intensive measure.

- A repeat staff Safety culture survey and Audit of DATIX incidents is recommended in 12 months.

\section{G406(P) IMPLEMENTATION OF A NEW PAEDIATRIC EARLY WARNING SYSTEM IN A TERTIARY PAEDIATRIC HOSPITAL}

H Ali, A Brough, E Lim, S Evans, AM Troy-Smith, J Mulholland, R Agbeko, M Thomas. Paediatrics, Great North Children's Hospital, Newcastle upon Tyne, UK

10.1136/archdischild-2018-rcpch.395

Paediatric Early Warning Systems (PEWS) aid timely recognition and response to a deteriorating child. In 2015 we identified that existing charts had substantial variability in use. Datix reporting and child death review panels identified avoidable harms resulting from inadequate monitoring and escalation.

Aims We aimed to design and implement a new PEWS following Kotter's 8-step change model to ensure inpatients have regular observations and an initial PEWS score within an hour of admission.

Methods The new PEWS charts were developed following focus groups with multidisciplinary staff. We sought management level support under the national 'Sign up to Safety' initiative. A regional forum was developed with the involvement of the Young People's Advisory Group and parents.

Changes included a new minimum set of observations, parental concern, colour coded scoring and escalation system. The charts were piloted initially in December 2015, and fully implemented to all paediatric wards in May 2016. Changes were tested using multiple PDSA cycles. Bimonthly audits were undertaken by the Clinical Governance and Risk Department (CGARD).

An education strategy included face-to-face teaching by designated PEWS champions, an e-learning module and in-situ simulation based teaching.

Results Audits demonstrated sustained improvement and compliance from December 2015 to June 2017 (table 1). Improvements were seen in measures including blood pressure and our new parental concern score.

Conclusion Sustained change has occurred through adaption and flexibility. The new chart is used across different local and regional sites, including intensive care.

\begin{tabular}{lccccc} 
Abstract G406(P) Table 1 & & \\
\hline & Observations & & \\
\hline \multicolumn{5}{c}{ Pre implementation } & Post implementation \\
\hline & Aug-15 & Dec-15 & Aug-16 & Dec-16 & Jun-17 \\
Parental Concern & & $52.63 \%$ & $58.33 \%$ & $69.23 \%$ & $80.0 \%$ \\
$\begin{array}{l}\text { Blood Pressure } \\
\text { All observations }\end{array}$ & $48.86 \%$ & $52.63 \%$ & $69.44 \%$ & $69.23 \%$ & $79.0 \%$ \\
recorded & $20.45 \%$ & $5.26 \%$ & $26.39 \%$ & $44.23 \%$ & $51.0 \%$ \\
& & & & & \\
\hline
\end{tabular}

Success was due to dedicated champions and senior nursing staff with support from the CGARD. Parents played a definitive role in shaping our work. Lessons included the need to develop a champions' network earlier and more frequent audit and feedback.

We aim to develop an electronic score, continue to evaluate the escalation pathways and review unplanned PICU admissions.

\section{G407(P) IMPROVING MANAGEMENT OF PROLONGED SEIZURES IN CHILDREN}

${ }^{1} \mathrm{~N}$ Bhangu, ${ }^{2} \mathrm{O}$ Shields, ${ }^{1} \mathrm{~K}$ Pryde. 'Paediatrics, Southampton Childrens Hospital, Southampton, UK; ${ }^{2}$ Anaesthetics, University Hospital Southampton, Southampton, UK

\subsection{6/archdischild-2018-rcpch.396}

Literature and local PICU case studies suggests high morbidity and mortality for children with prolonged seizures. A 6 month retrospective audit at a busy district general hospital in 2016 supported this and provided the basis for a regional project run jointly by paediatrics and anaesthetics.

A multiprofessional prospective quality improvement project was launched regionally in December 2016, to run throughout 2017. The aim is to improve morbidity and mortality of children with prolonged seizures as measured by:

1. Time to seizure termination

2. Percentage intubated, ventilated or admitted to level 3 care.

A secondary aim is to reduce the numbers with evidence of respiratory, haemodynamic or metabolic compromise.

Data is being collected via paper and online forms. Regional resources e.g. the online PICU regional guidleines have been utilised to prompt data collection. Local teams have been recruited at 7 hospitals within the region and results fed back on a 2 monthly basis.

Results have been collated regionally for time to seizure termination and time to phenytoin load. Other factors investigated are number of benzodiazepine doses given, proportion intubated and ventilated, duration of intubation and place of highest acuity of care.

Over the first 9 months of the project, 134 cases have been captured regionally. Initial data collection was slow and strategies to improve this including refinement of an electronic form, local leads championing the project and local targeted strategies such as (P)s and paper lists have been successful.

The first improvement is being implemented - focus on 'thinking ahead' to the next step of the treatment algorithm. Regionally this is planned via the PICU guidelines. Locally, 
run charts have been produced and local centres are considering how to best achieve this improvement.

The next stages involve identifying and implementing further improvements both locally and regionally. We are hoping to utilise a nurse or allied healthcare professional via the regional paediatric network to facilitate this. For all interventions, the PDSA cycles with continuous monitoring of outcomes will be used to track effects. Where local changes lead to improvement in outcomes, we will use the regional nature of the project to enable other centres to learn and benefit from this learning.

\section{G408(P) THE PRODUCTION OF A DIABETES INFORMATION FILM, BY YOUNG PEOPLE WHO HAVE DIABETES, AS A MEANS OF EDUCATING OTHERS}

${ }^{1}$ VE Dublon, ${ }^{2} \mathrm{~S}$ Green, ${ }^{1} \mathrm{M}$ Benitez-Castillo, ${ }^{3} \mathrm{~T}$ Edwards, ${ }^{4} \mathrm{~A}$ Leiva. ${ }^{1}$ Child Health, Royal Free Hospital, London, UK; ${ }^{2}$ Hospital School, Royal Free Hospital, London, UK; ${ }^{3}$ Child Health, Royal Free Hospital, London, UK; ${ }^{4}$ E17 Puppet Projet, London, UK

\subsection{6/archdischild-2018-rcpch.397}

Method After establishing a group of regular attendees to our Diabetes Education Days we wanted to make these more patient centred. We obtained a grant from the Diabetes Research and Wellness Foundation. Through the medium of puppet making and learning film skills the young people felt empowered producing their own educational film. Throughout the process, of four sessions spread over four weeks, the young people were supported by a multidisciplinary team consisting of consultant paediatrician, a specialist diabetes nurse, the deputy head of the Hospital school, a dietician, a team of professional puppet makers, and a film making expert. In addition to filming the actual process, the participants wrote and filmed their individual screenplays. There was opportunity to explore individual experiences and knowledge of diabetes in a fun, non-judgemental setting. Full consent was given by all attendees regarding the final distribution and use of the film.

Results The first of four sessions was a resounding success. Young people were desperate to attend the following sessions, altering schedules to ensure they could make them. During the process it became very clear they would like the film to be used as an educational tool. The response to the first viewing has given great verbal feedback and we are now in the process of collating formal qualitative data from the following groups: Children at the Royal Free Hospital School without diabetes, those with a new diagnosis and those who already have diabetes, which will be ready to be shared at the conference.

Conclusion Young people with diabetes embraced these sessions with huge enthusiasm. Having previously not known each other they quickly formed friendships and all actively took part, encouraging each other. They expressed and explored their knowledge in a relaxed environment, very different from clinic; given a rare and valuable opportunity to fully voice their opinions. At the same time, they loved the idea that the film productions they contributed to may go on to help other people.

For the professionals it was an incredibly inspiring experience and the film really does show diabetes through the eyes of the young people themselves.
G409(P) SAFETY CLIMATE \& CULTURE A SURVEY OF PAEDIATRIC STAFF IN DISTRICT GENERAL HOSPITAL

S Kamath, A Khan. Paediatrics, Daisy Hill Hospital, Southern Health and Social Care Trust, Newry, UK

\subsection{6/archdischild-2018-rcpch.398}

Background Organisations working towards a culture of safety need a reliable measure to monitor the success of their initiatives. Using Safety survey tool, an organisation can gain information about the perceptions of front-line clinical staff.

Aim To gain information about safety in the clinical area and management's commitment to safety.The survey also provides information about how perceptions vary across Multi-disciplinary teams (MDT).

Method QI Working group carried out Literature search for safety climate and culture.RCPCH -Safety survey was selected. Survey was pretested with a small group to ensure easy understanding and relevance.Survey was completed by MDT, medical, nursing, administrative and health care assistants in the Paediatric department.

Results 38 completed surveys received.Survey looked at Patient safety, Leadership, Staffing and communication.About $72 \%$ agreed that concerns were listened to by senior management. $76 \%$ agreeing that systems were in place to address concerns. $74 \%$ agreed that there was a medical and nursing leadership drive towards a safety centred organisation.Discussing errors at work was deemed easy. $72 \%$ felt that medical errors were handled appropriately. $65 \%$ received appropriate feedback about performance.

Conclusion This survey provided us in-depth information of safety culture within the department. It provided insight regarding various MDT's safety perception. We plan to address issues about error discussion. The Safety Awareness For Everyone i.e. SAFE initiative aims to reduce harm and improve safety.

\section{REFERENCE}

1. Safety Climate Survey. http://primaris.org/sites/default/files/resources/Patient\% 20Safety/safety\%20climate\%20survey.pdf

\section{G410(P) SHOULD ACCIDENTAL ADMINISTRATION OF RESIDUAL ANAESTHETIC AGENTS IN INTRAVENOUS LINES IN CHILDREN BE CLASSED A 'NEVER EVENT'?}

${ }^{1,2} \mathrm{C}$ Kanaris. 'North West and North Wales Paediatric Transport Service, Warrington, UK; ${ }^{2}$ The Centre for Social Ethics and Policy, The University of Manchester, Manchester, UK

\subsection{6/archdischild-2018-rcpch.399}

General surgical and paediatric teams are increasingly required to share the responsibility of the postoperative care of children undergoing routine surgical procedures, they are therefore the first to be called should a child become unwell in the immediate postoperative period. With this in mind it is imperative to raise awareness of potentially catastrophic consequences following the administration of residual neuromuscular blocking agents that may linger in the intravenous line after the patients have returned from theatre.

Incident reports of patients accidentally receiving residual neuromuscular blocking drug in the intravenous line following an operation may be rare but it is a clinical problem with potentially grave sequelae and one not currently classed as a 'never event' 